\title{
Syntheses of Concave-Shaped [5,5,5]-Tricyclic Triquinanes by Pd-Catalyzed Enediyne Cycloreduction
}

\author{
Chang Ho Oh, ${ }^{*}$ Mira Kim, and Chul Yun Rhim \\ Department of Chemistry, Hanvang Lniversity, Seoul 133-791, Korea. 'E-mail: changhoahanang.ackr \\ Received December 11, 2006
}

Key Words : Palladium. Catalỵst. Enediỵne. Cỵcloreduction

Pd-catalyzed enediyne cyclization gives a various polycyclic compounds in a very convenient single step. A few years ago, we reported a cascade cycloreduction of various enediynes leading to $[\mathrm{m}, 5 . \mathrm{n}]$-tricyclic compounds catalyzed by palladiun catalysts ${ }^{1}$ and, last year. we could synthesize Ceratopicanol by using this method as a key step. ${ }^{2}$ This reaction proceeded with high levels of stereoselectivities leading to concave-shaped triquinane skeletons accompanying a siguificant increase in structural conplexity.

A major problem was found to arise from competition between $\beta$-elinination of the alkylpalladium intermediates $A$ and carbopalladation of intermediate $B$ which formed $t$. respectively (Scheme 1). ${ }^{3}$ This problem could be overcome by changing the reaction conditions associated with mainly palladiun cataly sts and additives. Herein we wish to report a general entry to concave-shaped [5.5,5]-triquinane skeletons by entoloying Pd-catalyzed enediyne cycloreduction methodolog:

\section{Results and Discussion}

First, various enediynes were prepared according to the well-known methods (eq. 1).

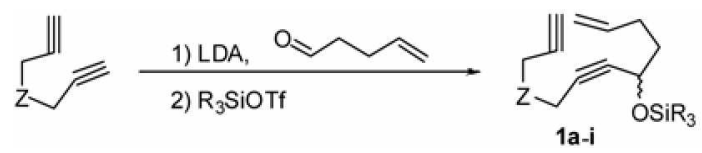

Deprotonation of diynes with $\mathrm{LDA}$ at $-78^{\circ} \mathrm{C}$ and then

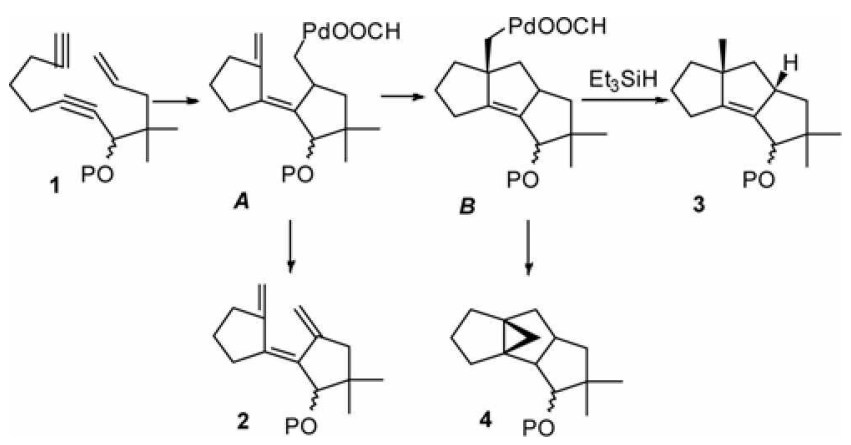

Scheme 1
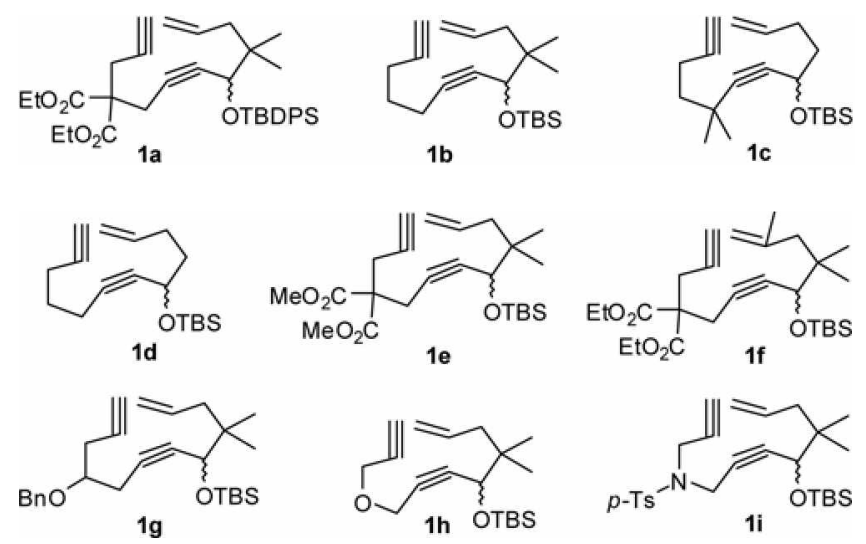

Figure 1

treatment of the corresponding aldehydes in THF gave the corresponding alcohols which were protected with trialkylsilyl trifluoromethanesulfonate to give our substrates 1a-i in g-scales (Figure 1). In order to find optimal cycloreduction conditions leading to a general entry to [5.5.5]-tricyclic compounds, we first tested a substrate 1a under different

Table 1. Cycloreduction of 1 a under Pd catalysis

\begin{tabular}{|c|c|c|c|c|}
\hline & $\begin{array}{l}\text { Pd catalyst (5 mol\%) } \\
\text { Additives (equiv) }\end{array}$ & Solvent & $\begin{array}{l}\text { Temp }\left({ }^{\circ} \mathrm{C}\right) \\
\text { lime }(\mathrm{h})\end{array}$ & $\begin{array}{c}\mathbf{3 a} \\
(\% \text { ield) }\end{array}$ \\
\hline 1 & $\begin{array}{l}{\left[(\pi \text {-allyl)PdCl }]_{2}\right.} \\
\mathrm{PPh}_{3}(0.1) \mathrm{HCOOH}(2)\end{array}$ & DMF & $80 / 2$ & 70 \\
\hline 2 & $\mathrm{Pd}(\mathrm{PhCN})_{2} \mathrm{Cl}_{2}$ & DMF & $80 / 6$ & 75 \\
\hline 3 & $\mathrm{Pd}\left(\mathrm{CH}_{3} \mathrm{CN}\right)_{2} \mathrm{Cl}_{2}$ & DMF & $80 / 8$ & $\mathrm{~N}_{\mathrm{I}}$ \\
\hline 4 & $\mathrm{Pd}_{2}\left(\mathrm{dba}_{3}\right.$ & DMF & $80 / 4$ & $58(2 a)$ \\
\hline 5 & $\mathrm{Pd}(\mathrm{OAc})_{2}$ & DMF & $80 / 4$ & Dimer \\
\hline 6 & $\mathrm{Pd}\left(\mathrm{PPh}_{3}\right)_{4}$ & DMF & $80 / 6$ & Dimer \\
\hline 7 & $2 \mathrm{~mol} \% \mathrm{Pd}(\mathrm{PhCN})_{2} \mathrm{Cl}_{2}$ & DMF & $100 / 4$ & 63 \\
\hline 8 & $\mathrm{Pd}(\mathrm{PhCN})_{2} \mathrm{Cl}_{2}$ & toluene & $110 / 3$ & 70 \\
\hline 9 & $\mathrm{Pd}(\mathrm{PhCN})_{2} \mathrm{Cl}_{2}$ & EDC & $70 / 2$ & 47 \\
\hline 10 & $\mathrm{Pd}(\mathrm{PhCN})_{2} \mathrm{Cl}_{2}$ & Diosane & $100 / 3$ & 59 \\
\hline 11 & $\mathrm{Pd}(\mathrm{PhCN})_{2} \mathrm{Cl}_{2}$ & $\mathrm{CH}_{3} \mathrm{CN}$ & $80 / 4$ & 68 \\
\hline 12 & $\begin{array}{l}\mathrm{Pd}(\mathrm{PhCN})_{2} \mathrm{Cl}_{2} \\
\mathrm{HCOOH}(2) / \mathrm{Et} t_{3} \mathrm{SiH}(2)\end{array}$ & $\begin{array}{l}\text { DMF or } \\
\text { diosane }\end{array}$ & $80 / 6$ & $72-78$ \\
\hline
\end{tabular}

Isolated vield of triene $\mathbf{2 a}$. 
conditions (Table 1).

We have utilized $[(\pi \text {-allyl }) \mathrm{PdCl}]_{2} \quad \mathrm{Pd}\left(\mathrm{CH}_{3} \mathrm{CN}_{2} \mathrm{Cl}_{2}\right.$. $\mathrm{Pd}_{2}(\mathrm{dba})_{3} . \mathrm{Pd}(\mathrm{OAc})_{2} \cdot \mathrm{Pd}\left(\mathrm{PPh}_{3}\right)_{4}$, and $\mathrm{Pd}(\mathrm{PhCN})_{2} \mathrm{Cl}_{2}$ as $\mathrm{Pd}-$ precatalysts for cycloreduction of 1a using two equivalent of $\mathrm{HCOOH}$ as a reductant and DMF as a solvent (entries 1-6). Among these. $[(\pi \text {-allyl }) \mathrm{PdCl}]_{2}$ and $\mathrm{Pd}(\mathrm{PhCN})=\mathrm{Cl}$ - successfully catalyzed 1 a to afford the corresponding product $3 a$ in $70 \%$ and $75 \%$ yields. respectively. $\mathrm{Pd}\left(\mathrm{CH}_{3} \mathrm{CN}\right)_{2} \mathrm{Cl}_{2}$. even similar to $\mathrm{Pd}(\mathrm{PhCN})=\mathrm{Cl}_{2}$. did not catalyze this reaction. $\mathrm{Pd}_{2}(\mathrm{dba})_{3}$ catalyzed this reaction but afforded to the triene compound $\mathbf{2 a}$ as a major product. Finally. when we employed $\mathrm{Pd}(\mathrm{OAc})_{2}$ and $\mathrm{Pd}\left(\mathrm{PPh}_{3}\right)_{4}$ as catalysts. the unexpected dimerized product was obtained exclusively. Completion of this sequential cyclization catalyzed by $\mathrm{Pd}$ compound required increased stability and fast reduction of the Pd-alkyl intermediate. The decreased amount of precatalyst from 5 $\mathrm{mol} \%$ to $2 \mathrm{~mol} \%$ was less effective in terms of isolated yield of 3 (entry 7). Use of nonpolar solvents such as toluene. EDC. dioxane and acetonitrile were inferior to DMF (entries 8-11). To facilitate formation of 3. stronger reductant might be required to cleave the carbon-Pd bond of $B$ as soon as it was formed. Otherwise triene 2 and further cyclized product 4 were formed. Triethylsilane turned out to be a good choice for this rapid reduction of alky lpalladium intermediate over unwanted $\beta$-elimination (entry 12). Thus. an optimal condition for this cycloreduction was found: 5 $\mathrm{mol}_{0} \mathrm{Pd}(\mathrm{PhCN})_{2} \mathrm{Cl}_{2} .2$ equivalents of $\mathrm{HCOOH}$ and $\mathrm{Et}_{3} \mathrm{SiH}$
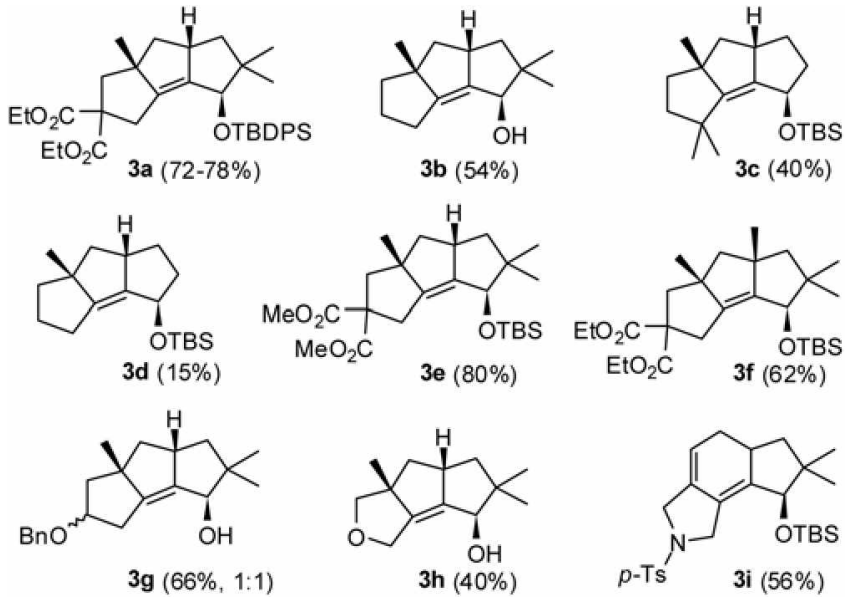

in DMF or dioxane. This condition was applied to the structurally diverse enediy'nes $\mathbf{1 b}-\mathbf{1 i}$ (Figure 2).

While $\mathbf{1 b}$ and $1 \mathrm{c}$ possessing a geminal dimethyl group gave the desired product $3 \mathrm{~b}$ and $3 \mathrm{c}$ in $54 \%$ and $40 \%$ yields. respectively. 1d with no geminal alkyl group was less reactive to give $3 \mathrm{~d}$ in only $15 \%$ yield. The substrate le has the same carbon skeleton as 1a but has different protecting groups. The cycloreduction of 1e was virtually similar to that of 1 a to give $3 \mathrm{e}$ in $80 \%$ yield. The $\mathbf{1 f}$, a homolog of 1 a having a methyl substituent on the olefinic position. also gave the cycloreduced product $3 \mathrm{f}$ in $62 \%$ yield under our conditions. The substrate $1 \mathrm{~g}$ was designed for application of its cycloreduced product. Cycloreduction of $\mathbf{1 g}$ under our conditions gave about $1: 1$ mixture of two isomeric products $3 \mathrm{~g}$ in $66 \%$ yield. Both isomers $3 \mathrm{~g}-1$ and $3 \mathrm{~g}-2$ could be separated by HPLC chromatography. Finally. this method was applied two enediynes bearing a heteroatom linker. The oxygen-linkered $1 \mathrm{~h}$ was also cycloreduced to give $3 \mathrm{~h}$ in $40 \%$ yield. while the nitrogen-linkered $1 \mathrm{i}$ was not cycloreduced to $3 \mathrm{i}$ but cycloisomerized to give $3 \mathrm{i}$ in $56 \%$ yield. Note that some products $\mathbf{3 b}$. $\mathbf{3 g}$ and $\mathbf{3 h}$ were desilylated by adding $1.0 \mathrm{M}$ solution of tetrabutylanmonium fluoride solution in THF.

Stereochemistries of $3 \mathrm{a}-\mathrm{h}$ were speculated based on $2 \mathrm{D}$ NMR of 3j-2 and X-ray' study of 6 . which were intermediates to the ceratopicanol synthesis (Scheme 2). ${ }^{2}$

A catalytic mixture of $[(\pi \text {-allyl }) \mathrm{PdCl}]_{2}\left(5 \mathrm{~mol}^{0}\right), \mathrm{PPl}_{3}(20$ mol\%). $\mathrm{HCOOH}(1.0$ eq). and triethylsilane (10 eq) in 1.4dioxane was found to transform enediyne $1 \mathbf{j}$ to the crcloreduced tricycle $3 \mathrm{j}$ in $70-75 \%$ yield $(\alpha / \beta$ ratio of angular $\mathrm{H}=$ $1 / 3$ ) along with only a little amount of the triene. ${ }^{4}$ Stereoselectivity in our cycloreduction was turned out that the present cycloreduction was highly stereoselective having concave-relationship among three fused 5-membered rings. Both $3 \mathrm{j}-1$ and $3 \mathrm{j}-2$ could be transformed to $( \pm)$-ceratopicanol.

In conclusion, we have shown a general entry to fused [5.5.5] tricyclic compounds with concave shapes starting from the corresponding linear enediynes substrates under mild Pd cataly sis."

\section{Experimentals}

General experimental procedure (3a-h): A mixture of

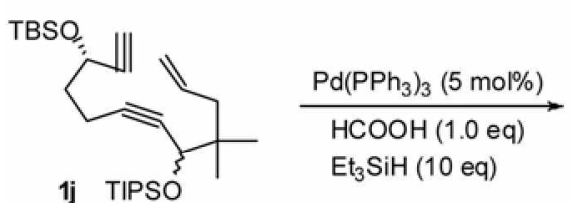

1j TIPSO

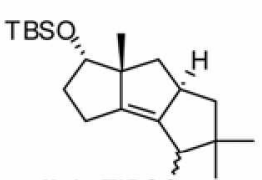

3j-1 TIPSO

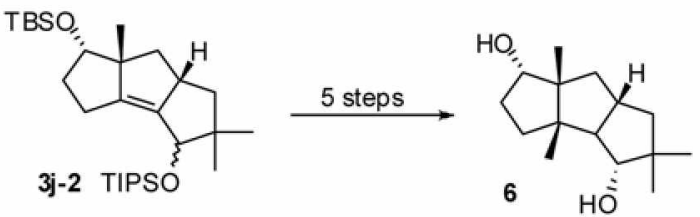

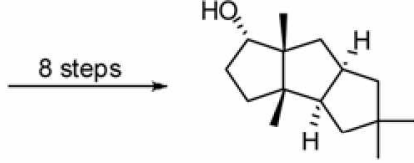

( \pm )-Ceratopicanol 
enediyne 1a-h $(0.4 \mathrm{mmol})$, Pd ( $5 \mathrm{~mol} \%), \mathrm{PPh}_{3}(10 \mathrm{~mol} \%)$. $\mathrm{HCOOH}(0.8 \mathrm{mmol})$. triethylsilane $(0.8 \mathrm{mmol})$ and dried solvent $(1.0 \mathrm{~mL})$ in a $5 \mathrm{~mL}$ test tube was heated at $80^{\circ} \mathrm{C}$ $100^{\circ} \mathrm{C}$ for $2-24 \mathrm{~h}$ under argon atmosphere. The reaction was monitored by checking TLC periodically. Upon completion. the solvent was removed under vacuum and the crude product was subjected for flash column chromatography to afford the corresponding products $3 \mathbf{a}-\mathbf{h}$ in fair to good yields as shown in Figure 2. 3a: IR $\left(\mathrm{NaCl} \mathrm{cm} \mathrm{cm}^{-1}\right)$ 2932. 2858, 1733 . 1472: ${ }^{1} \mathrm{H} \mathrm{NMR}\left(400 \mathrm{MHz} . \mathrm{CDCl}_{3}\right) \delta 7.68-7.64(\mathrm{~m} .4 \mathrm{H})$, 7.44-7.31 (m. 6H) . 4.20-3.94 (m. 4H). 3.85 (s. 1H). $3.54(\mathrm{~m}$. lH) $2.19(\mathrm{~d} . J=13.6 \mathrm{~Hz}, 1 \mathrm{H}), 2.18(\mathrm{dd} J=16.4 .1 .6 \mathrm{~Hz}$. lH). 2.13 (dd. $J=12.0 .4 .4 \mathrm{~Hz} .1 \mathrm{H}) .2 .06(\mathrm{dd} . J=11.6 .6 .4$ Hz. $1 \mathrm{H}) .1 .93(\mathrm{~d} . J=13.6 \mathrm{~Hz} .1 \mathrm{H}) .1 .87$ (dd. $J=11.2 .2 .0$ $\mathrm{Hz}, 1 \mathrm{H}) .1 .43(\mathrm{dd} . J=11.2,9.2 \mathrm{~Hz}, 1 \mathrm{H}) .1 .21$ (t. $J=7.2 \mathrm{~Hz}$. $3 \mathrm{H}) .1 .14$ (s. $3 \mathrm{H}), 1.12$ (t. $J=7.2 \mathrm{~Hz}, 3 \mathrm{H}) .1 .04$ (s. 9H). 1.00 (dd. $J=11.2 .4 .6 \mathrm{~Hz}, 1 \mathrm{H}$ ) 0.90 (s. $3 \mathrm{H}$ ) 0.75 (s. $3 \mathrm{H}$ ): ${ }^{13} \mathrm{C}$ NMR $\left(100 \mathrm{MHz} . \mathrm{CDCl}_{3}\right) \delta 172.21 .172 .12 .145 .04 .141 .70$. 136.00, 134.43, 134.22, 129.37. 129.25, 127.43, 127.21 . $77.13,61.57,61.34,61.30,59.04,52.62,50.23,46.53$. $46.26,42.94,31.54,28.65,26.98,24.36,23.77,19.57,14.01$. 13.90: HRMS calculated for $\mathrm{C}_{36} \mathrm{H}_{48} \mathrm{O}_{5} \mathrm{SiNa}^{+} 611.3169$; found. 611.3165. 3b: IR $\left(\mathrm{NaCl} \mathrm{cm}^{-1}\right) 3356,2949,286 \mathrm{l}:{ }^{1} \mathrm{H}$ NMR $\left(400 \mathrm{MHz}, \mathrm{CDCl}_{3}\right) \delta 3.92$ (s. $\left.1 \mathrm{H}\right), 3.63(\mathrm{~m}, 1 \mathrm{H}) .2 .24-2.13$ (m. $2 \mathrm{H}) .2 .10(\mathrm{dd} . J=12.0 .6 .4 \mathrm{~Hz}, \mathrm{lH}) .2 .05-1.97(\mathrm{~m} . \mathrm{lH})$. $1.92-1.83(\mathrm{~m} .1 \mathrm{H}) .1 .76(\mathrm{dd} J=12.8 .9 .2 \mathrm{~Hz} .1 \mathrm{H}) .1 .58-1.53$ (m. 1H) $1.42-1.37$ (m, 3H) 1.12 (s. 3H) 1.05 (s. 3H) 1.03 (dd. $J=12.4 .6 .8 \mathrm{~Hz} .1 \mathrm{H}$ ). 0.96 (s. $3 \mathrm{H}):{ }^{13} \mathrm{C}$ NMR $(100$ $\mathrm{MHz} . \mathrm{CDCl}_{3}$ ): $\delta 151.75 .139 .69 .76 .61 .60,60,51.93 .50 .58$. $46.25,44.11,39.48,28.70,26.49,23.05,22.62,22.39$ : HRMS calculated for $\mathrm{C}_{14} \mathrm{H}_{22} \mathrm{ONa}^{+} 229.1568$; found. 229.1559. 3c: IR (NaCl. cm $\left.{ }^{-1}\right) 2955,2931.2859,1472 ;{ }^{1} \mathrm{H}$ NMR (400 $\left.\mathrm{MHz} . \mathrm{CDCl}_{3}\right) \delta 4.71(\mathrm{t} . J=5.6 \mathrm{~Hz} . \mathrm{lH}) .3 .5 \mathrm{l}(\mathrm{m} . \mathrm{lH}) .2 .13$ $2.06(\mathrm{~m} .1 \mathrm{H}) .2 .00-1.87(\mathrm{~m} .3 \mathrm{H}) .1 .78-1.66(\mathrm{~m} .2 \mathrm{H}) .1 .53-$ 1.43 (m. 2H). 1.19 (s. 6H). 1.16 (s. 3H) $1.03-0.97$ (m. 2H). 0.88 (s. $9 \mathrm{H}$ ). 0.08 (s. 3H) 0.07 (s. $3 \mathrm{H}):{ }^{13} \mathrm{C} \mathrm{NMR}(100 \mathrm{MHz}$. $\left.\mathrm{CDCl}_{3}\right) \delta 155.69,139.86,68.26 .63 .39,52.56 .51 .44,44.43$. $39.91,38.35,36.83,30.08,29.82 .27 .77,25.94,23.25$. 18.00, -4.09. -4.37: HRMS calculated for $\mathrm{C}_{2 j} \mathrm{H}_{36} \mathrm{OSiNa}^{+}$ 343.2433; found. 343.2429 . 3d: ${ }^{1} \mathrm{H} \mathrm{NMR}\left(400 \mathrm{MHz}, \mathrm{CDCl}_{3}\right)$ $\delta 4.28(\mathrm{t}, J=4.8 \mathrm{~Hz}, \mathrm{lH}), 2.46(\mathrm{~m}, \mathrm{lH}) .2 .22-2.14(\mathrm{~m} .3 \mathrm{H})$. 2.06-1.94 (m. 4H) $1.84-1.74(\mathrm{~m} .3 \mathrm{H}), 1.60-1.56(\mathrm{~m} .1 \mathrm{H})$. 1.26 (s. 3H). $1.13-1.06$ (m. 1H). 0.83 (s. 9H). 0.04 (s. 3H). 0.03 (s. $3 \mathrm{H}$ ): HRMS calculated for $\mathrm{C}_{18} \mathrm{H}_{32} \mathrm{OSiNa}^{+} 315.2120$ : found. 315.2126. 3e: IR $\left.(\mathrm{NaCl} \mathrm{cm})^{-1}\right) 2954.2930,2895$. 2858, 1738. 1435; ${ }^{1} \mathrm{H}$ NMR ( $\left.400 \mathrm{MHz}, \mathrm{CDCl}_{3}\right) \delta 3.75$ (s. $3 \mathrm{H}) .3 .7 \mathrm{l}(\mathrm{s} . \mathrm{HH}) .3 .69(\mathrm{~s}, 3 \mathrm{H}) .3 .50(\mathrm{~m} . \mathrm{HH}), 2.86(\mathrm{~m} .2 \mathrm{H})$, $2.37($ d. $J=14.0 \mathrm{~Hz}, 1 \mathrm{H}) .2 .12(\mathrm{dd} . J=11.2 .6 .0 \mathrm{~Hz}, 1 \mathrm{H})$. $2.08(\mathrm{~d} . J=13.6 \mathrm{~Hz}, \mathrm{lH}) .1 .76(\mathrm{dd} . J=12.8 .10 .0 \mathrm{~Hz}, 1 \mathrm{H})$. 1.51 (dd. $J=11.2 .9 .6 \mathrm{~Hz} .1 \mathrm{H}$ ). 1.11 (s. $3 \mathrm{H}$ ). 1.00 (s. $3 \mathrm{H}$ ). 0.96 (dd $J=8.8 .4 .6 \mathrm{~Hz} .1 \mathrm{H}$ ), 0.86 (s. $9 \mathrm{H}$ ). 0.80 (s. $3 \mathrm{H}$ ). 0.04 (s. $3 \mathrm{H}) .0 .00$ (s. $3 \mathrm{H}$ ): ${ }^{13} \mathrm{C}$ NMR $\left(100 \mathrm{MHz} . \mathrm{CDCl}_{3}\right) \delta$ 172.76. 172.61. 143.57. 143.27. 76.37, 61.78, 59.11, 52.85. $52.78,50.19 .46 .44,46.18,42.59 .32 .17,28.67,26.14$. $25.77,23.88,23.73,18.20,-4.33,-5.07$ : HRMS calculated for: $\mathrm{C}_{24} \mathrm{H}_{4}\left(\mathrm{O} \mathrm{O}_{\leq} \mathrm{SiNa}^{-}{ }^{4} 59.2543\right.$; found. 459.2548 . 3f: IR
$\left(\mathrm{NaCl} . \mathrm{cm}^{-1}\right) 2956,2930,2858,1735,1475:{ }^{1} \mathrm{H}$ NMR (400 $\left.\mathrm{MHz} . \mathrm{CDCl}_{3}\right) \delta 4.26-4.1 \mathrm{l}(\mathrm{m}, 4 \mathrm{H}) .3 .69$ (s. IH). 2.69 (d. $J=$ $16.4 \mathrm{~Hz}, 1 \mathrm{H}), 2.79(\mathrm{~d} . J=16.4 \mathrm{~Hz}, 1 \mathrm{H}) .2 .32(\mathrm{~d} . J=13.6$ Hz. lH). 2.10 (d. $J=13.6 \mathrm{~Hz}, \mathrm{lH}$ ). 1.89 (s. $2 \mathrm{H}$ ). $1.49-1.46$ (m. 2H). 1.35 (s. 3H), 1.28-1.24 (m. 6H), 1.20 (s. 3H). 1.02 (s. 3H). 0.88 (s. 9H). 0.80 (s. 3H), 0.05 (s. 3H). 0.01 (s. 3H): ${ }^{13} \mathrm{C}$ NMR (100 MHz. $\left.\mathrm{CDCl}_{3}\right) \delta 172.68,172.46 .145 .48$, $143.33,77.13,61.63,61.57,61.40 .59 .92,59.06,58.15$. 53.78, 49.06, 45.42, 31.56, 31.46, 29.69, 29.22. 25.83. 24.70, 18.24, 14.03. 13.98, -4.30, -5.21: HRMS calculated for $\mathrm{C}_{27} \mathrm{H}_{4} \mathrm{O}_{5} \mathrm{SiNa}^{-}$501.3012; found. 501.3018. 3g-1: IR $\left(\mathrm{NaCl} . \mathrm{cm}^{-1}\right) 3419,3031,2927,2862,1454:{ }^{1} \mathrm{H}$ NMR (400 $\left.\mathrm{MHz}, \mathrm{CDCl}_{\mathrm{j}}\right) \delta 7.36-7.27(\mathrm{~m} .5 \mathrm{H}), 4.49(\mathrm{~s}, 2 \mathrm{H}) .4 .14-4.08$ (m. lH). $3.91(\mathrm{~d}, J=4.4 \mathrm{~Hz}, 1 \mathrm{H}) .3 .51(\mathrm{~m}, 1 \mathrm{H}) .2 .70$ (ddd. $J$ $=15.4,6.4 .1 .2 \mathrm{~Hz}, \mathrm{lH}) .2 .25(\mathrm{dt} . J=16.4 .5 .4 \mathrm{~Hz}, 1 \mathrm{H}) .2 .13$ (dd. $J=11.6 .6 .4 \mathrm{~Hz}, 1 \mathrm{H}), 1.82-1.72(\mathrm{~m} .2 \mathrm{H}) .1 .71(\mathrm{~d} . J=$ $4.4 \mathrm{~Hz}, 1 \mathrm{H}$ ). 1.35 (dd. $J=11.6,6.4 \mathrm{~Hz}, 2 \mathrm{H}$ ), 1.26 (s. $3 \mathrm{H}$ ). 1.05 (s. 3H), 1.00 (dd. $J=12.4 .6 .8 \mathrm{~Hz} .1 \mathrm{H}$ ). 0.93 (s. 3H): ${ }^{13} \mathrm{C}$ NMR $\left(100 \mathrm{MHz} . \mathrm{CDCl}_{3}\right) \delta 147.64,142.29 .138 .59$. 128.34. 127.57. 127.46. 81.42. 76.40. 71.08. 58.10.53.03. $48.87,46.18,45.74,43.84,31.47,28.70,25.02,22.57$ : HRMS calculated for $\mathrm{C}_{21} \mathrm{H}_{28} \mathrm{O}_{2} \mathrm{~K}^{-}$351.1726: found. 351.1728. 3g-2: IR $\left(\mathrm{NaCl} . \mathrm{cm}^{-1}\right) 3419.2960,2861,1715$. 1496: ${ }^{1} \mathrm{H}$ NMR $\left(400 \mathrm{MHz}, \mathrm{CDCl}_{3}\right) \delta 7.36-7.28(\mathrm{~m}, 5 \mathrm{H})$. 4.48 (ABq. $\Delta \delta=4.4 \mathrm{~Hz} . J=12.0 \mathrm{~Hz}, 2 \mathrm{H}), 4.38(\mathrm{~m}, \mathrm{lH})$, 3.95 (s. $1 \mathrm{H}$ ). 3.59 (m. 1H). 2.56 (ddd. $J=17.2 .8 .0 .4 .4 \mathrm{~Hz}$. $1 \mathrm{H}$ ), 2.33 (ddd, $J=17.2 .4 .8 .2 .4 \mathrm{~Hz}, 1 \mathrm{H}$ ). 2.09 (dd. $J=11.6$. $6.0 \mathrm{~Hz}, 1 \mathrm{H}), 2.02(\mathrm{dd} . J=12.8 .6 .4 \mathrm{~Hz}, 1 \mathrm{H}), 1.77(\mathrm{dd} . J=$ $12.8 .8 .8 \mathrm{~Hz} .1 \mathrm{H}) .1 .52(\mathrm{dd} . J=11.6 .6 .0 \mathrm{~Hz}, 1 \mathrm{H}) .1 .34(\mathrm{~m}$. $1 \mathrm{H}) .1 .25$ (s. $1 \mathrm{H}) .1 .10$ (s. $3 \mathrm{H}) .1 .04$ (s. 3H). $1.07-1.02(\mathrm{~m}$. 1H). 0.99 (s. $3 \mathrm{H}):{ }^{13} \mathrm{C}$ NMR $\left(100 \mathrm{MHz} . \mathrm{CDCl}_{3}\right) \delta 147.88$, $141.46,138.52$. 128.33, 127.61, 127.48. 82.34, 76.57, 71.12. 59.30, 52.03, 49.74, 46.87, 46.45, 44.04. 30.17, 28.61. 23.93. 22.60: HRMS calculated for $\mathrm{C}_{23} \mathrm{H}_{28} \mathrm{O}_{2} \mathrm{Na}^{-} 335.1987$; found. 335.1991 . 3h: IR $\left(\mathrm{NaCl} \mathrm{cm}^{-1}\right) 3419,2952,2862$. 1456: ' $\mathrm{H}$ NMR $\left(400 \mathrm{MHz}, \mathrm{CDCl}_{3}\right) \delta 4.32-4.22(\mathrm{~m}, 2 \mathrm{H})$. $3.94(\mathrm{~d} . J=4.8 \mathrm{~Hz}, 1 \mathrm{H}) .3 .81(\mathrm{~m}, 1 \mathrm{H}) .3 .71(\mathrm{~d} . J=8.0 \mathrm{~Hz}$. $1 \mathrm{H}) .3 .39$ (d. $J=8.0 \mathrm{~Hz}, \mathrm{lH}$ ). 2.06 (dd. $J=11.6 .6 .0 \mathrm{~Hz}$. $1 \mathrm{H}), 1.81$ (dd. $J=12.8 .9 .2 \mathrm{~Hz}, 1 \mathrm{H}), 1.54$ (dd. $J=11.6 .9 .2$ $\mathrm{Hz}, 1 \mathrm{H}), 1.43(\mathrm{~d} . J=4.8 \mathrm{~Hz}, 1 \mathrm{H}), 1.28$ (s. $3 \mathrm{H}), 1.11$ (dd. $J=$ $12.8,7.2 \mathrm{~Hz}, 1 \mathrm{H}$ ). 1.06 (s. 3H), 1.01 (s. $3 \mathrm{H}$ ): ${ }^{13} \mathrm{C}$ NMR (100 $\left.\mathrm{MHz}, \mathrm{CDCl}_{3}\right) \delta 146.56,141.28,79.39,76.22,63.20,61.67$. $52.55,47.65,46.68,43.54,28.53$. 22.41. 21.64; HRMS calculated for $\mathrm{C}_{12} \mathrm{H}_{21} \mathrm{O}_{2} \mathrm{~K}^{-}$247.1100: found. 247.1114. 3i: IR $\left(\mathrm{NaCl} \mathrm{cm}^{-1}\right)$ 2956. 2929. 2857. 1347; ${ }^{1} \mathrm{H}$ NMR $(400 \mathrm{MHz}$. $\left.\mathrm{CDCl}_{3}\right) \delta 7.77-7.70(\mathrm{~m} .2 \mathrm{H}), 7.32-7.29(\mathrm{~m} .2 \mathrm{H}), 4.31(\mathrm{bs}$, lH), 4.24-3.97 (m, 4H). 2.43 (s. $3 \mathrm{H}), 2.26-2.14$ (m. $5 \mathrm{H}$ ), 2.02 (d. $J=16.0 \mathrm{~Hz}, 1 \mathrm{H}$ ). 1.07 (s. 3H). 0.94 (s. 3H). 0.91 (s. 9H). 0.10 (s. 3H). 0.08 (s. 3H): ${ }^{13} \mathrm{C}$ NMR $\left(100 \mathrm{MHz} . \mathrm{CDCl}_{3}\right)$ $\delta 143.23$. 138.49, 134.27, 130.87, 129.68. 127.91. 127.54, $127.25,84.13,56.05,54.31 .49 .27 .42 .93 .28 .39,26.19$. $24.24,23.93,21.49,21.26,18.27,-2.63,-4.12$ : HRMS calculated for $\mathrm{C}_{26} \mathrm{H}_{3} 9 \mathrm{NO}_{3} \mathrm{SSiNa}^{-} 496.2318$; found. 496.2322 .

Acknowledgments. We wish to acknowledge the funancial support of Korea Research Foundation (KRF). 


\section{References}

1. (a) Oh. C. H.: Rhim. C. Y: Kang. J. H.: Kim. A.: Park. B. S.: Seo, Y. Tetrahedron Lett 1996, 37. 8875. (b) Oh, C. H. Rhim. C. Y.: Jung, H. H. Jung, S. H. Bull. Korean Chem. Soc. 1999. 20. 643 .

2. (a) Oh, C. H.: Rhim. C. Y.: Kim, M.: Park. D. I.: Gupta, A. K. Sylett 2005. 2694.

3. Similar types of alkylpalladium intermediates bearing a $\beta-$ hydrogen are prone to undergo elimination to the corresponding alkenes. For reviews, see (a) Trost, B. M. Science 1991, 254. 1471. (b) Trost. B. M. Acc. Chem. Res. 1990, 23, 34. (c) Trost, B.
M; Shi. Y. J. Am. Chem Soc. 1993, 115. 12491

4. For the use of formic acid or formates as a hydrogen donor in palladium-mediated reactions. see: (a) Tsuji. J. Palladim Reagents and Catahysts: Wiley: Chichester. England. 1995. (b) Tsuji. T.: Mandai. T. Symthesis 1996. 1. (c) Trost. B. M. Li, Y.J.Am. Chem. Soc, 1996. 118,6625 . (d) Oh, C. H.: Jung, H. H: sung. H. R.; Kim. J. D. Tetrahedron 2001, 57, 1723. (e) Oh. C. H.; Park. S. J. Tetrahedron Lett. 2003. H. 3785 .

5. (a) Co. T. T.: Shim. S. C.: Cho. C. S.: Kim. D.-U.: Kim. T.-T. Bull. Chent. Korean Soc. 2005. 26. 1359. (b) Park. C. M.: Hant. S. Y.: Seo. N. Y.; Byu, C.-H.: Gyoung. Y. S. Bull. Chem. Korean Soc. 2006. 27,1727 . 